\title{
Current Concepts for Patellar Dislocation
}

\author{
Maximilian Petri ${ }^{1,}$; Max Ettinger ${ }^{2}$; Timo Stuebig ${ }^{1}$; Stephan Brand ${ }^{1}$; Christian Krettek ${ }^{1}$; \\ Michael Jagodzinski ${ }^{3}$; Mohamed Omar ${ }^{1}$ \\ ${ }^{1}$ Department of Trauma, Hannover Medical School, Hannover, Germany \\ ${ }_{2}^{2}$ Department of Orthopaedic Surgery, Hannover Medical School, Hannover, Germany \\ 3 Department of Orthopaedic Surgery, Agaplesion Ev. Hospital Bethel, Buckeburg, Germany \\ ${ }^{*}$ Corresponding author: Maximilian Petri, Department of Trauma, Hannover Medical School, Hannover, Germany. Tel: +49-5115322050, Fax: $+49-5115325877$, \\ E-mail: petri.maximilian@mh-hannover.de
}

Received: April 29, 2015; Accepted: May 11, 2015

\begin{abstract}
Context: Patellar dislocation usually occurs to the lateral side, leading to ruptures of the Medial Patellofemoral Ligament (MPFL) in about $90 \%$ of the cases. Even though several prognostic factors are identified for patellofemoral instability after patellar dislocation so far, the appropriate therapy remains a controversial issue.

Evidence Acquisition: Authors searched the Medline library for studies on both surgical and conservative treatment for patellar dislocation and patellofemoral instability. Additionally, the reference list of each article was searched for additional studies.

Results: A thorough analysis of the anatomical risk factors with a particular focus on patella alta, increased Tibial Tuberosity-Trochlear Groove(TT-TG) distance, trochlear dysplasia as well as torsional abnormalities should be performed early after the first dislocation to allow adequate patient counseling. Summarizing the results of all published randomized clinical trials and comparing surgical and conservative treatment after the first-time patellar dislocation until today indicated no significant evident difference for children, adolescents, and adults. Therefore, nonoperative treatment was indicated after a first-time patellar dislocation in the vast majority of patients.

Conclusions: Surgical treatment for patellar dislocation is indicated primarily in case of relevant concomitant injuries such as osteochondral fractures, and secondarily for recurrent dislocations.
\end{abstract}

Keywords:Knee; Patella; Patellar Dislocation; Evidence-Based Medicine; Medial Patellofemoral Ligament; Patellofemoral Instability

\section{Context}

Patellar dislocations account for $2 \%$ - $3 \%$ of the knee joint injuries $(1,2)$. It is the most common cause of traumatic hemarthrosis in children, and the second most common in adolescents after ruptures of the anterior cruciate ligament (3). The patella is usually dislocated laterally, and subsequently causing ruptures of the Medial Patellofemoral Ligament (MPFL) in about $90 \%$ of the patients $(1,4)$. Patellar dislocation mainly affects young and active people with an almost equal distribution between male and female and a peak age of 10 - 20 years (2, 5-10). Most of the first-time patellar dislocations occur during sport activities, with reported incidence of $55 \%$ - $72 \%(2,5,11,12)$. Stability of the patellofemoral joint is maintained by the complex interaction of three stabilizing groups: static stabilizers (patellofemoral joint geometry), active (M. quadriceps femoris) and passive soft tissue stabilizers (retinacular ligaments). These stabilizers contribute in various extents to patellofemoral joint stability through the range of knee flexion $(13,14)$.

\section{Evidence Acquisition}

A search of the Medline library for studies on patellar dislocation and patellofemoral instability surgical and conservative treatments was performed in April 2015. Additionally, the reference list of each article was searched for additional studies of interest. Studies were included if they either compared surgical and conservative treatments of patellar dislocations, or provided information on diagnostic tools, surgical techniques and outcomes of patellofemoral joint instability.

\section{Results}

3.1. Study Background for Operative vs. Nonoperative Treatment of the First-Time Patellar Dislocation

Prognostic factors for patellofemoral instability and patient satisfaction after either surgical or conservative treatment following first-time patellar dislocation remain a matter of debate. However, there is a consensus regarding the indication of surgical treatment in case of relevant concomitant injuries such as osteochondral fractures $(6,9)$. Furthermore, patella alta, increased Tibial Tuberosity-Trochlear Groove (TT-TG) distance, trochlear

Copyright (c) 2015, Kashan University of Medical Sciences. This is an open-access article distributed under the terms of the Creative Commons Attribution-NonCommercial 4.0 International License (http://creativecommons.org/licenses/by-nc/4.0/) which permits copy and redistribute the material just in noncommercial usages, provided the original work is properly cited. 
dysplasia as well as torsional abnormalities are suggested as prognostic factors for recurrent dislocation (15).

To date, a total of nine prospective randomized clinical trials, comparing conservative and surgical treatment after first-time patellar dislocation, were performed, reporting inconsistent results (7, 16-23). Especially limited number of the patients reduces the validity of the studies.

Sillanpaa et al. conducted two studies in a military hospital, both on over $90 \%$ of male patients $(21,22)$. The results revealed lower rates of redislocation after surgical treatment in one study (22), and equal redislocation rates but better regain of preinjury activity after surgery in the other one (21). Neither study showed clear subjective benefits in the long-term follow-up compared to conservative treatment.

Nikku et al. (18) reported the seven-year follow-up results of 127 patients after first-time patellar dislocation. In the conservative group, redislocations occurred in $39 \%$ of the patients, compared to $31 \%$ in the operative group. In the conservative group, the mean Kujala score was 90 - 94 points, and $81 \%$ of the patients were satisfied with their result. In the operative group, the mean Kujala score was 88 - 89 points, and only $67 \%$ of the patients considered their result satisfactory (18). The same research group had previously reported the two-year-results of this trial, showing no benefit from surgery compared to conservative treatment (19). As a conclusion, the authors did not recommend routine surgery after first-time patellar dislocation.

In 2008, Christiansen et al. (7) randomized 80 patients with primary patellar dislocation into either conservative treatment or operative treatment by reinsertion of the MPFL to the adductor tubercle. Their two-year followup revealed a mean Kujala score of 78 and a $20 \%$ redislocation rate in the conservative group, compared to a mean Kujala score of 85 and a $17 \%$ redislocation rate in the operative group (7).

Palmu et al. (20) randomized 74 children and adolescents less than 16 years old. A clinical follow-up was performed after two years, and subjective phone-call questionnaires were completed after a mean of 6 and 14 years. This is the longest follow-up so far reported in the literature. In the conservative group, $75 \%$ of the subjects considered their result good or excellent, compared to $66 \%$ in the operative group. Redislocation rates were remarkably high in both groups (71\% and $67 \%$ in the conservative and operative groups, respectively). As the only significant predictor of the long term outcome, a positive family history of patellar instability could be determined. Therefore, the authors voted against routine repair after patellar dislocation (20).

\subsection{Indications for Nonoperative and Operative Treatment}

A systematic review of 70 studies by Stefancin and Parker (6) recommended non-operative treatment for the majority of patients after first-time patellar dislocation, with the exceptions of concomitant chondral and osteochondral fractures, and furthermore, somewhat blurred, exceptions for extended medial soft-tissue damage and strong lateralization of the patella. The meta-analysis by Smith et al. (24) revealed a tendency towards lower redislocation rates, but also higher rates of patellofemoral osteoarthritis after surgical treatment.

The most recent Cochrane Review (25) investigating surgical versus non-surgical treatment for patellar dislocation included five studies with a total of 339 patients and a varying follow-up of 2 - 7 years. All studies were criticized for methodological flaws. The authors concluded that there is insufficient evidence for any clinical outcomes difference between surgical or non-surgical treatment for primary patellar dislocation, and no evidence concerning recurrent patellar dislocation. They therefore recommended adequately powered randomized controlled multi-centric trials according to the contemporary standards (25).

Most recently, a systematic review of four overlapping meta-analyses by Erickson et al. (26) found that operative treatment of acute patellar dislocations may result in a lower rate of recurrent dislocations than non-operative treatment ( $24.0 \%$ vs. $34.6 \%)$, but did not improve functional outcome scores.

Regarding recurrent patellar dislocation and chronic patellofemoral instability, there are no randomized clinical trials at all.

To summarize, non-operative treatment is indicated after a first-time patellar dislocation in a vast majority of patients. Surgical treatment is indicated primarily in case of relevant concomitant injuries such as osteochondral fractures, and secondarily for recurrent dislocations.

\subsection{Magnetic Resonance Imaging Analysis}

A thorough analysis of the anatomical risk factors, usually by MRI, is conducted after the first dislocation. Children and adolescents may sometimes not be fully aware of having sustained a patellar dislocation $(2,27)$. Typical findings in the MRI are joint effusion, contusions of the cartilage on the lateral femoral condyle and the medial patella, chondral or osteochondral fragments, and a torn medial retinaculum and MPFL $(2,28)$. The evaluation of the MRI should include the following factors (13).

\subsubsection{Sulcus Angle}

Sulcus angle was measured on transverse MRIs according to van Huyssteen et al. (29) (Figure 1).

The average normal sulcus angle is approximately $138 \pm$ $6^{\circ}$. A sulcus angle larger than $145^{\circ}$ is an indicator of trochlear dysplasia (30-32).

\subsubsection{Dysplasia of the Trochlea}

Trochlear dysplasia is measured according to the classification of Dejour et al. (31) and Dejour and Saggin (33). 


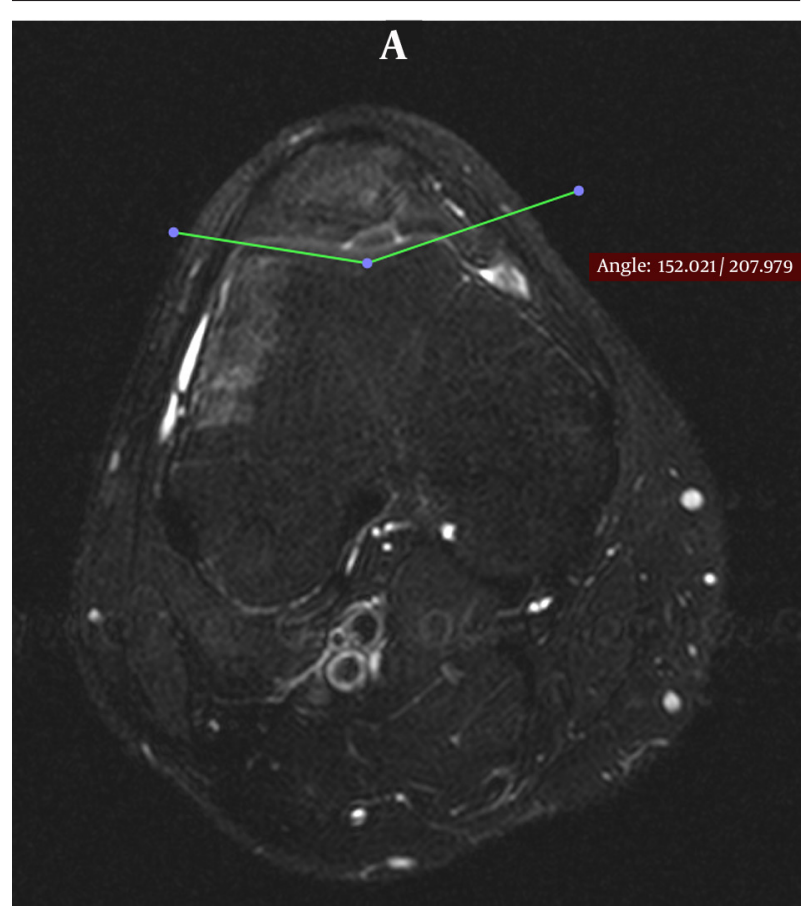

Figure 1. Measurement of the Sulcus Angle to Determine Trochlear Dysplasia on Axial Magnetic Resonance Imaging Slice (T2)

Trochlear dysplasia is categorized according to the above mentioned studies into A (fairly shallow trochlea), B (flat or convex trochlea), C (asymmetry of the trochlea facets: lateral trochlea convex, medial facet hypoplastic), and D (asymmetry of the trochlea facets, vertical joint and cliff pattern). On a true lateral X-ray, the crossing sign, which is a line represented by the deepest part of the trochlear groove crossing the anterior aspect of the condyles, is an indicator of trochlear dysplasia $(30,31,34)$. Increased trochlear dysplasia is described as a risk factor for patellar instability $(6,35)$. The shape of the trochlear groove and degree of trochlea dysplasia are considered similar in pediatrics and adults (2).

\subsubsection{Depth and Facet Asymmetry of the Trochlea}

It is measured on transverse MRIs according to Pfirrmann et al. (36). Patients with higher-type trochlear dysplasia or asymmetry are described to benefit from a surgical management not only addressing the soft tissue, but also including bony corrections such as the medialization of the tibial tubercle or trochleoplasty $(37,38)$.

\subsubsection{The Insall-Salvati Index}

Determination of the patellar height is a ratio of the patellar tendon length to the longest sagittal dimension of the patella on sagittal T1-weighted images $(39,40)$ (Figure 2). An Insall-Salvati index larger than 1.2 indicates a patella alta, and an index smaller than 0.8 indicates a patella baja (41).

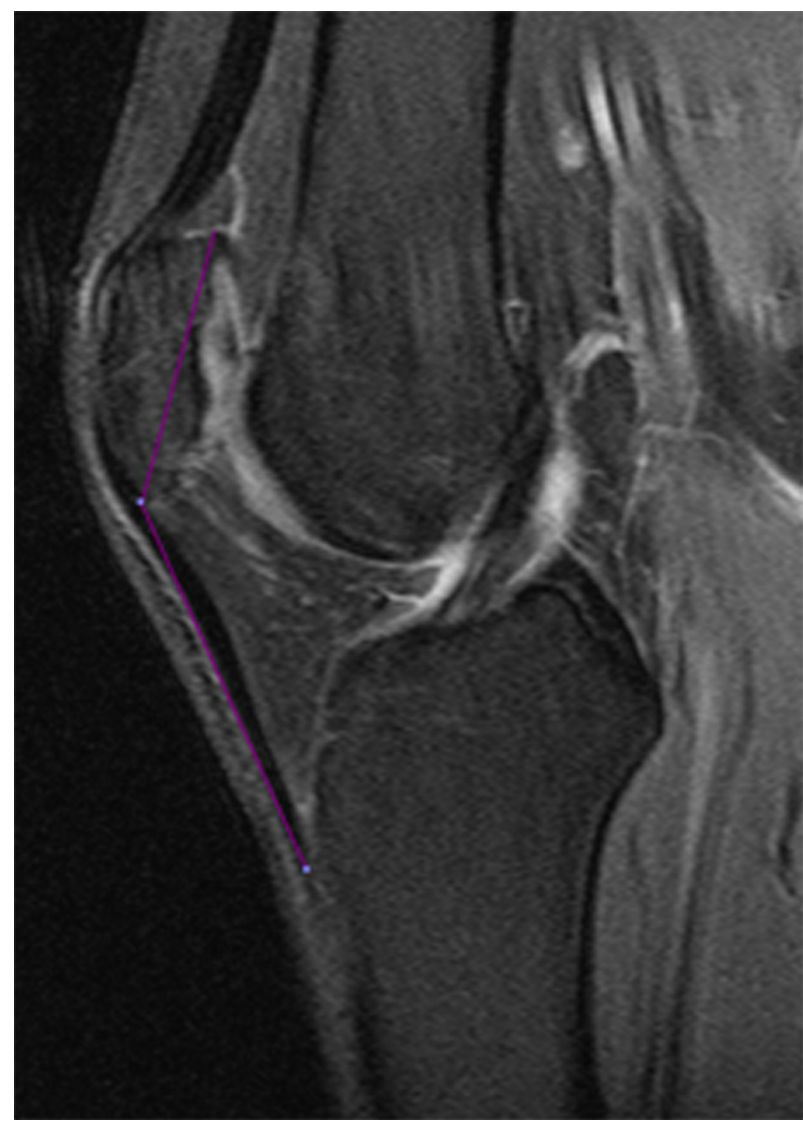

Figure 2. Measurement of the Insall-Salvati Index to Determine a Patella Alta on a Sagittal MRI Slice (T2)

\subsubsection{Tibial Tuberosity-Trochlear Groove Distance}

It is measured on transverse CT or MRI slices according to Schoettle et al. (42) (Figures 3 and 4). The transverse CTor MRI-slices at both the position of the tibial tuberosity (TT) and the trochlear groove (TG) are cross-sectioned, and the distance between the TT and the TG is measured. Dejour et al. (31) suggested a threshold of $20 \mathrm{~mm}$ for a pathologically increased TT-TG in 1994, while following authors suggested to lower the limit to $15 \mathrm{~mm}$ as an indication of tubercle realignment surgery in symptomatic patients $(35,43)$.

\subsubsection{Rupture Patterns of the Medial Patellofemoral Ligament}

It is determined on transverse images according to the method introduced in previous studies $(2,44,45)$. It distinguishes between complete and incomplete ruptures as well as patellar based (Figure 5), femoral based (Figure 6) intraligamentary or mid-substance (Figure 7), and combined ruptures. Ruptures of the MPFL can be complete or partial, with an about equal distribution described in the literature $(13,45,46)$. Since decision-making also depends on the kind of MPFL injury, the identification of the rupture site by MRI is of particular importance (2). 


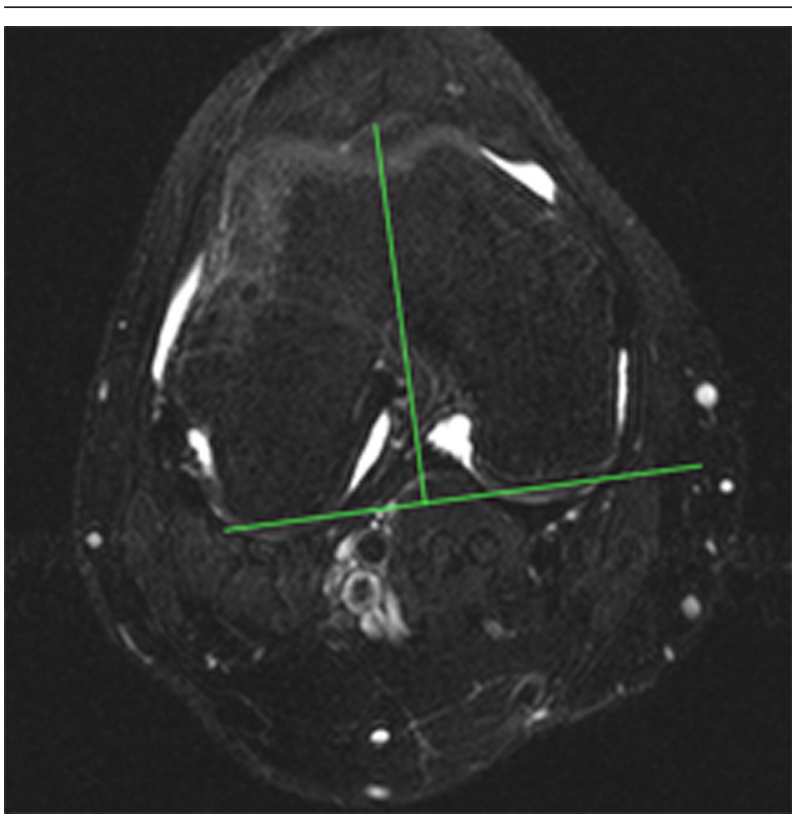

Figure 3. Measurement of the Tibial Tuberosity-Trochlear Groove Distance on the Femoral Axial Magnetic Resonance Imaging Slice (T2)

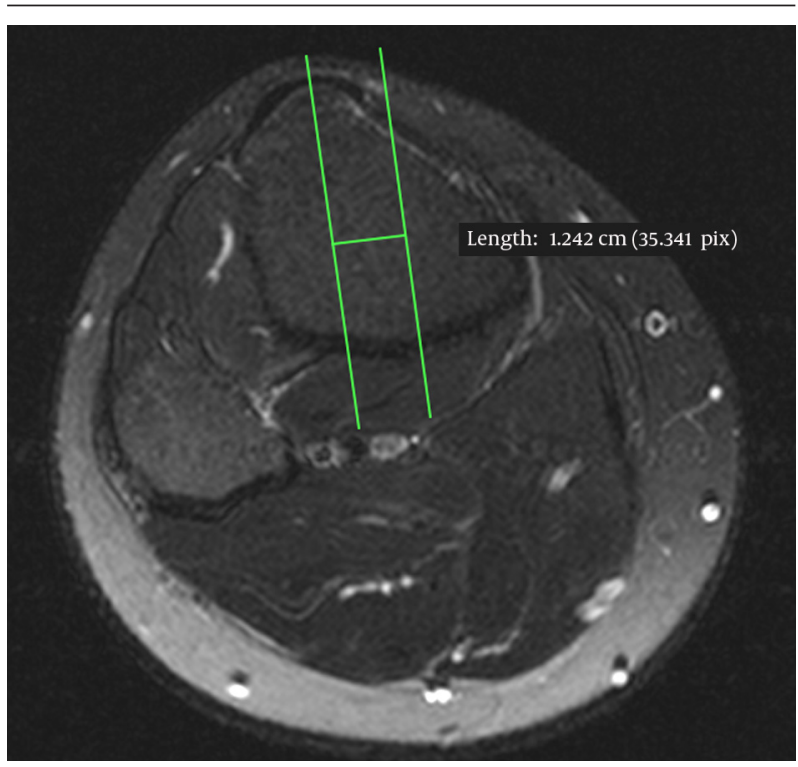

Figure 4. Measurement of the Tibial Tuberosity-Trochlear Groove Distance on the Tibial Axial Magnetic Resonance Imaging Slice (T2)

Since the particular risk factors for recurrent dislocations are patella alta, increased TT-TG-distance, and trochlear dysplasia as well as torsional abnormalities (15), they should be analyzed early after the first dislocation to allow adequate patient counseling.

\subsection{Surgical Treatment Options}

There is no level I data regarding indications of the different surgical treatment options. Traditionally, a reefing suture of the medial patellofemoral soft-tissues combined

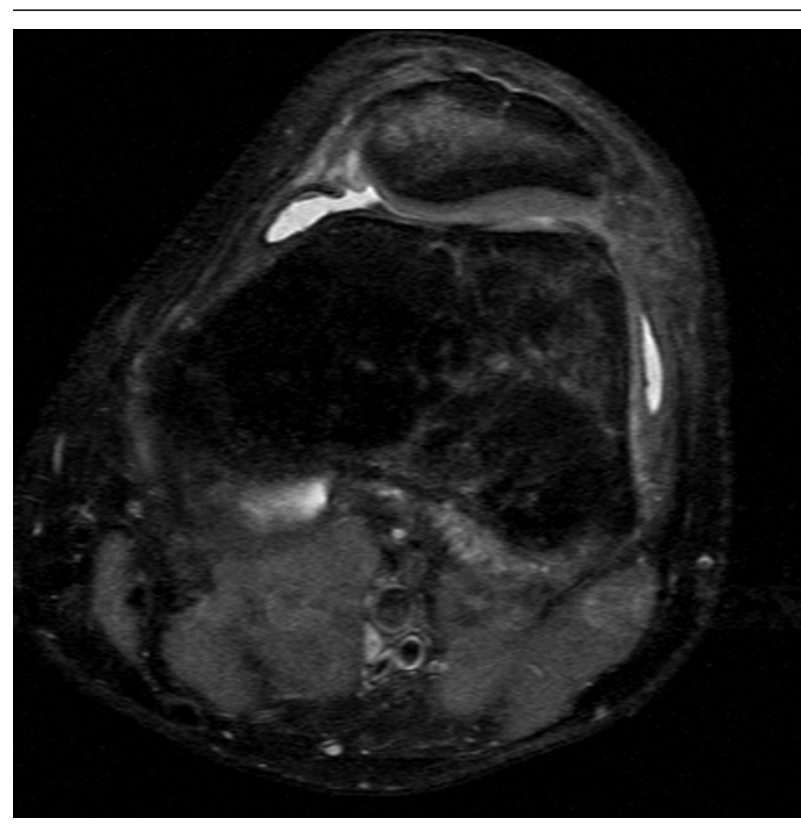

Figure 5. Patellar Based Rupture of the Medial Patellofemoral Ligament on Axial Magnetic Resonance Imaging Slice (T2)

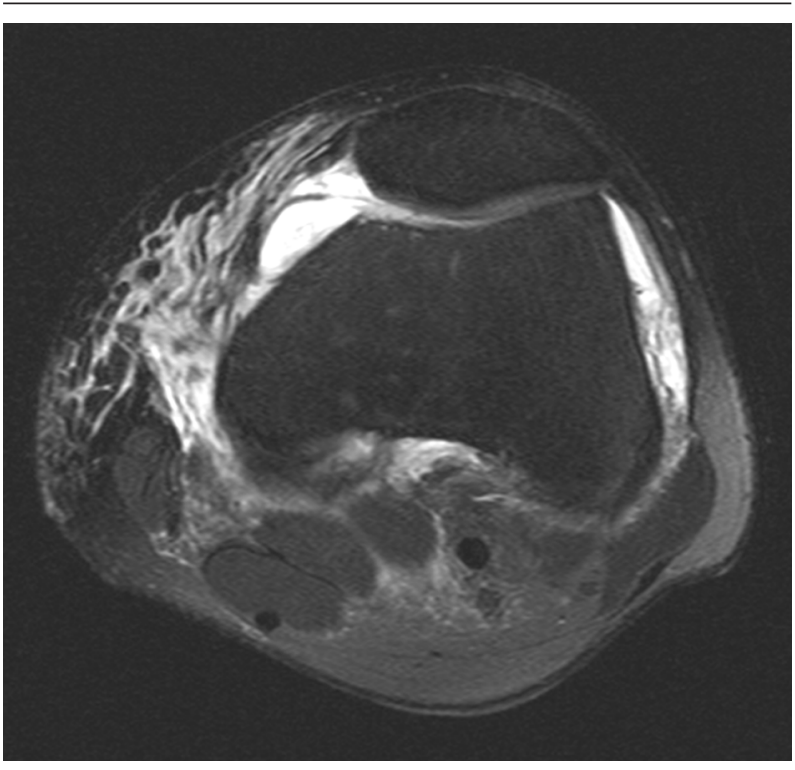

Figure 6. Femoral Based Rupture of the Medial Patellofemoral Ligament on Axial Magnetic Resonance Imaging Slice (T2)

with a lateral release has been performed as the standard procedure for many years. Since lateral release creates a risk for secondary medial patellofemoral instability (47), nowadays it is only rarely indicated anymore.

\subsection{Medial Patellofemoral Ligament Repair or Re- construction}

Recently, a variety of techniques with promising shortterm results are suggested for reconstruction of the MPFL. 


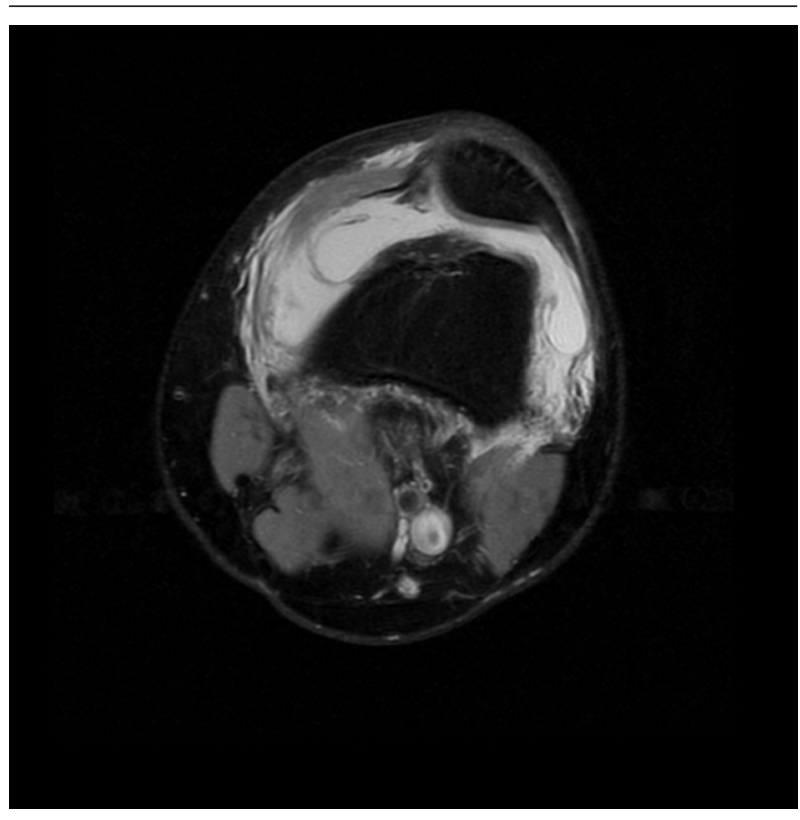

Figure 7. Complete Intraligamentary (Mid-Substance) Rupture of the Medial Patellofemoral Ligament on Axial Magnetic Resonance Imaging Slice (T2)

MPFL repair or reconstruction is indicated in the presence of a physiological TT-TG distance $(<15 \mathrm{~mm})(48)$, and only mild trochlear dysplasia (type A/B according to Dejour et al. (48)). Depending on the rupture site (13), refixation is either performed with suture anchors for femoral-based or patellar-based ruptures (Figures 5 and 6 ), or arthroscopic suture for intraligamentary/mid-substance ruptures (Figure 7). Repair instead of reconstruction is especially indicated for children and adolescents with open physes, as the femoral insertion of the MPFL is located in the area of the distal medial femoral epiphysis.

MPFL reconstruction can be performed with either a gracilis (49-52) or semitendinosus tendon (53-55), quadriceps tendon graft (56), or patellar tendon graft (16). The transfer of the distal adductor magnus tendon is advocated by some authors to avoid donor site morbidity $(57,58)$

The recent study by Bitar et al. (16) represents the first prospective randomized clinical trial comparing MPFL reconstruction with conservative management. They reported a significantly better mean of final Kujala score in the surgical group (88.9 points) than in the non-operative group (70.8 points). Consequently, the surgical group presented a significantly higher percentage of 'good/excellent' results (71.43\%) compared to the non-operative group (25.0\%). In the non-operative group, 35\% of the patients sustained recurrent dislocations or subluxations, whereas no recurrences or subluxations were reported in the surgical group.

However, these results should be interpreted cautiously, since the study only included a total of 41 knees and a two-year follow-up. The role of medial reconstruction should be evaluated carefully in the light of different risk factors (children, valgus knee, trochlear dysplasia etc.).

\subsection{Tibial Tuberosity Transfer and Trochleoplasty}

The transfer of the tibial tuberosity represents an advancement of the Trillat procedure (59). It is indicated in the presence of a pathologically increased TT-TG distance $(>15 / 18 / 20$ $\mathrm{mm})(48,60)$. Usually a combined distalization and medialization of the tuberosity is performed after a coronary osteotomy, with subsequent screw osteosynthesis.

Trochleoplasty is a highly invasive surgical procedure which deepens and remodels the trochlear groove to allow improved patellar tracking. This procedure is indicated in the presence of high-level trochlear dysplasia (types B - D based on Dejour et al. $(33,48)$ ) and intact patellofemoral joint cartilage as a last resort. It is usually combined with MPFL reconstruction. The postoperative rehabilitation is protracted and the risk of patellofemoral osteoarthritis is potentially increased $(33,38)$. However, with recent improvements of surgical techniques, results are satisfactory to restore patellar stability and improved clinical scores and no patellofemoral osteoarthritis at a mean seven-year follow-up (61).

\section{Conclusions}

Summarizing the results of all published randomized clinical trials and comparing surgical and conservative treatment after first-time patellar dislocation until today indicated no evident significant difference between children, adolescents, and adults.

A thorough analysis of the anatomical risk factors with a particular focus on patella alta, increased TT-TG-distance, and trochlear dysplasia as well as torsional abnormalities should be performed early after the first dislocation.

Surgical treatment is primarily indicated in case of relevant concomitant injuries such as osteochondral fractures, and secondarily for recurrent dislocations. Complications almost exclusively occur after surgical treatment. Even though there still is a lack of evidence, there is a strong tendency towards conservative treatment after a first-time patellar dislocation. The advantages and disadvantages of different therapeutic strategies should be brought out to the patient.

\section{Authors' Contributions}

Study concept and design: Maximilian Petri, Max Ettinger, Timo Stuebig, Stephan Brand, Michael Jagodzinski, Mohamed Omar; acquisition of data: Maximilian Petri, Max Ettinger, Timo Stuebig, Stephan Brand, Mohamed Omar; analysis and interpretation of data: Maximilian Petri, Max Ettinger, Timo Stuebig, Stephan Brand, Mohamed Omar; drafting of the manuscript: Maximilian Petri, Michael Jagodzinski, Mohamed Omar; critical revision of the manuscript for important intellectual content: Max Ettinger, Timo Stuebig, Stephan Brand, Christian Krettek, Michael Jagodzinski; statistical analysis and administrative, technical, and material support: Max Ettinger, and Christian Krettek, Michael Jagodzinski; study supervision: Michael Jagodzinski and Christian Krettek. 


\section{References}

1. Arendt EA, Fithian DC, Cohen E. Current concepts of lateral patella dislocation. Clin Sports Med. 2002;21(3):499-519.

2. Balcarek P, Walde TA, Frosch S, Schuttrumpf JP, Wachowski MM, Sturmer KM, et al. Patellar dislocations in children, adolescents and adults: a comparative MRI study of medial patellofemoral ligament injury patterns and trochlear groove anatomy. Eur J Radiol. 2011;79(3):415-20.

3. Abbasi D, May MM, Wall EJ, Chan G, Parikh SN. MRI findings in adolescent patients with acute traumatic knee hemarthrosis. $J$ Pediatr Orthop. 2012;32(8):760-4.

4. Burks RT, Desio SM, Bachus KN, Tyson L, Springer K. Biomechanical evaluation of lateral patellar dislocations. Am J Knee Surg. 1998;11(1):24-31.

5. Atkin DM, Fithian DC, Marangi KS, Stone ML, Dobson BE, Mendelsohn C. Characteristics of patients with primary acute lateral patellar dislocation and their recovery within the first 6 months of injury. Am J Sports Med. 2000;28(4):472-9.

6. Stefancin JJ, Parker RD. First-time traumatic patellar dislocation: a systematic review. Clin Orthop Relat Res. 2007;455:93-101.

7. Christiansen SE, Jakobsen BW, Lund B, Lind M. Isolated repair of the medial patellofemoral ligament in primary dislocation of the patella: a prospective randomized study. Arthroscopy. 2008;24(8):881-7.

8. Kujala UM, Jaakkola LH, Koskinen SK, Taimela S, Hurme M, Nelimarkka O. Scoring of patellofemoral disorders. Arthroscopy. 1993;9(2):159-63.

9. Oestern S, Varoga D, Lippross S, Kaschwich M, Finn J, Buddrus B, et al. [Patella dislocation]. Unfallchirurg. 2011;114(4):345-58.

10. Ostermeier S, Holst M, Bohnsack M, Hurschler C, StukenborgColsman C, Wirth CJ. In vitro measurement of patellar kinematics following reconstruction of the medial patellofemoral ligament. Knee Surg Sports Traumatol Arthrosc. 2007;15(3):276-85.

11. Fithian DC, Paxton EW, Stone ML, Silva P, Davis DK, Elias DA, et al Epidemiology and natural history of acute patellar dislocation. Am J Sports Med. 2004;32(5):1114-21.

12. Nietosvaara Y, Aalto K, Kallio PE. Acute patellar dislocation in children: incidence and associated osteochondral fractures. $J$ Pediatr Orthop. 1994;14(4):513-5.

13. Petri M, von Falck C, Broese M, Liodakis E, Balcarek P, Niemeyer $\mathrm{P}$, et al. Influence of rupture patterns of the medial patellofemoral ligament (MPFL) on the outcome after operative treatment of traumatic patellar dislocation. Knee Surg Sports Traumatol Arthrosc. 2013;21(3):683-9.

14. Senavongse W, Amis AA. The effects of articular, retinacular, or muscular deficiencies on patellofemoral joint stability: a biomechanical study in vitro. J Bone Joint Surg Br. 2005;87(4):577-82.

15. Steensen RN, Bentley JC, Trinh TQ, Backes JR, Wiltfong RE. The prevalence and combined prevalences of anatomic factors associated with recurrent patellar dislocation: a magnetic resonance imaging study. Am J Sports Med. 2015;43(4):921-7.

16. Bitar AC, Demange MK, D'Elia CO, Camanho GL. Traumatic patellar dislocation: nonoperative treatment compared with MPFL reconstruction using patellar tendon. Am J Sports Med. 2012;40(1):114-22.

17. Camanho GL, Viegas Ade C, Bitar AC, Demange MK, Hernandez AJ. Conservative versus surgical treatment for repair of the medial patellofemoral ligament in acute dislocations of the patella. Arthroscopy. 2009;25(6):620-5.

18. Nikku R, Nietosvaara Y, Aalto K, Kallio PE. Operative treatment of primary patellar dislocation does not improve medium-term outcome: A 7-year follow-up report and risk analysis of 127 randomized patients. Acta Orthop. 2005;76(5):699-704.

19. Nikku R, Nietosvaara Y, Kallio PE, Aalto K, Michelsson JE. Operative versus closed treatment of primary dislocation of the patella. Similar 2-year results in 125 randomized patients. Acta Orthop Scand. 1997;68(5):419-23.

20. Palmu S, Kallio PE, Donell ST, Helenius I, Nietosvaara Y. Acute patellar dislocation in children and adolescents: a randomized clinical trial. J Bone Joint Surg Am. 2008;90(3):463-70.

21. Sillanpaa PJ, Maenpaa HM, Mattila VM, Visuri T, Pihlajamaki H. Arthroscopic surgery for primary traumatic patellar disloca- tion: a prospective, nonrandomized study comparing patients treated with and without acute arthroscopic stabilization with a median 7-year follow-up. Am J Sports Med. 2008;36(12):2301-9.

22. Sillanpaa PJ, Mattila VM, Maenpaa H, Kiuru M, Visuri T, Pihlajamaki $\mathrm{H}$. Treatment with and without initial stabilizing surgery for primary traumatic patellar dislocation. A prospective randomized study. J Bone Joint Surg Am. 2009;91(2):263-73.

23. Petri M, Liodakis E, Hofmeister M, Despang FJ, Maier M, Balcarek $\mathrm{P}$, et al. Operative vs conservative treatment of traumatic patellar dislocation: results of a prospective randomized controlled clinical trial. Arch Orthop Trauma Surg. 2013;133(2):209-13.

24. Smith TO, Song F, Donell ST, Hing CB. Operative versus non-operative management of patellar dislocation. A meta-analysis. Knee Surg Sports Traumatol Arthrosc. 2011;19(6):988-98.

25. Hing CB, Smith TO, Donell S, Song F. Surgical versus non-surgical interventions for treating patellar dislocation. Cochrane Database Syst Rev. 2011;(11):CD008106.

26. Erickson BJ, Mascarenhas R, Sayegh ET, Saltzman B, Verma NN Bush-Joseph CA, et al. Does Operative Treatment of First-Time Patellar Dislocations Lead to Increased Patellofemoral Stability? A Systematic Review of Overlapping Meta-analyses. Arthroscopy. 2015;31(6):1207-15.

27. Kirsch MD, Fitzgerald SW, Friedman H, Rogers LF. Transient lateral patellar dislocation: diagnosis with MR imaging. AJR Am J Roentgenol. 1993;161(1):109-13.

28. Spritzer CE, Courneya DL, Burk DJ, Garrett WE, Strong JA. Medial retinacular complex injury in acute patellar dislocation: MR findings and surgical implications. AJR Am J Roentgenol. 1997;168(1):117-22.

29. van Huyssteen AL, Hendrix MR, Barnett AJ, Wakeley CJ, Eldridge JD. Cartilage-bone mismatch in the dysplastic trochlea. An MRI study. J Bone Joint Surg Br. 2006;88(5):688-91.

30. Colvin AC, West RV. Patellar instability. J Bone Joint Surg Am. 2008;90(12):2751-62.

31. Dejour H, Walch G, Nove-Josserand L, Guier C. Factors of patellar instability: an anatomic radiographic study. Knee Surg Sports Traumatol Arthrosc. 1994;2(1):19-26.

32. Merchant AC, Mercer RL, Jacobsen RH, Cool CR. Roentgenographic analysis of patellofemoral congruence. J Bone Joint Surg Am. 1974;56(7):1391-6.

33. Dejour D, Saggin P. The sulcus deepening trochleoplasty-the Lyon's procedure. Int Orthop. 2010;34(2):311-6.

34. Weber-Spickschen TS, Spang J, Kohn L, Imhoff AB, Schottle PB. The relationship between trochlear dysplasia and medial patellofemoral ligament rupture location after patellar dislocation: an MRI evaluation. Knee. 2011;18(3):185-8.

35. Balcarek P, Jung K, Ammon J, Walde TA, Frosch S, Schuttrumpf JP, et al. Anatomy of lateral patellar instability: trochlear dysplasia and tibial tubercle-trochlear groove distance is more pronounced in women who dislocate the patella. Am J Sports Med. 2010;38(11):2320-7.

36. Pfirrmann CW, Zanetti M, Romero J, Hodler J. Femoral trochlear dysplasia: MR findings. Radiology. 2000;216(3):858-64.

37. Verdonk R, Jansegers E, Stuyts B. Trochleoplasty in dysplastic knee trochlea. Knee Surg Sports Traumatol Arthrosc. 2005;13(7):529-33.

38. Schottle PB, Fucentese SF, Pfirrmann C, Bereiter H, Romero J Trochleaplasty for patellar instability due to trochlear dysplasia: A minimum 2-year clinical and radiological follow-up of 19 knees. Acta Orthop. 2005;76(5):693-8.

39. Insall J, Salvati E. Patella position in the normal knee joint. Radiology. 1971;101(1):101-4.

40. Miller TT, Staron RB, Feldman F. Patellar height on sagittal MR imaging of the knee. AJR Am J Roentgenol.1996;167(2):339-41.

41. Carson WJ, James SL, Larson RL, Singer KM, Winternitz WW. Patellofemoral disorders: physical and radiographic evaluation. Part II: Radiographic examination. Clin Orthop Relat Res. 1984;(185):178-86.

42. Schoettle PB, Zanetti M, Seifert B, Pfirrmann CW, Fucentese SF, Romero J. The tibial tuberosity-trochlear groove distance; a comparative study between CT and MRI scanning. Knee. 2006;13(1):26-31. 
43. Koeter S, Diks MJ, Anderson PG, Wymenga AB. A modified tibial tubercle osteotomy for patellar maltracking: results at two years. J Bone Joint Surg Br. 2007;89(2):180-5.

44. Elias DA, White LM, Fithian DC. Acute lateral patellar dislocation at MR imaging: injury patterns of medial patellar soft-tissue restraints and osteochondral injuries of the inferomedial patella. Radiology. 2002;225(3):736-43.

45. Balcarek P, Ammon J, Frosch S, Walde TA, Schuttrumpf JP, Ferlemann KG, et al. Magnetic resonance imaging characteristics of the medial patellofemoral ligament lesion in acute lateral patellar dislocations considering trochlear dysplasia, patella alta, and tibial tuberosity-trochlear groove distance. Arthroscopy. 2010;26(7):926-35.

46. Sanders TG, Morrison WB, Singleton BA, Miller MD, Cornum KG. Medial patellofemoral ligament injury following acute transient dislocation of the patella: MR findings with surgical correlation in 14 patients.J Comput Assist Tomogr. 2001;25(6):957-62.

47. Song GY, Hong L, Zhang H, Zhang J, Li Y, Feng H. Iatrogenic medial patellar instability following lateral retinacular release of the knee joint. Knee Surg Sports Traumatol Arthrosc. 2015

48. Dejour H, Walch G, Neyret P, Adeleine P. [Dysplasia of the femoral trochlea]. Rev Chir Orthop Reparatrice Appar Mot. 1990;76(1):45-54.

49. Christiansen SE, Jacobsen BW, Lund B, Lind M. Reconstruction of the medial patellofemoral ligament with gracilis tendon autograft in transverse patellar drill holes. Arthroscopy. 2008;24(1):82-7.

50. Quirbach S, Smekal V, Rosenberger RE, El Attal R, Schottle PB. [Anatomical double-bundle reconstruction of the medial patellofemoral ligament with a gracilis autograft]. Oper Orthop Traumatol. 2012;24(2):131-9.

51. Schottle PB, Romero J, Schmeling A, Weiler A. Technical note: anatomical reconstruction of the medial patellofemoral ligament using a free gracilis autograft. Arch Orthop Trauma Surg. 2008;128(5):479-84.

52. Becher C, Kley K, Lobenhoffer P, Ezechieli M, Smith T, Ostermeier $S$. Dynamic versus static reconstruction of the medial patello- femoral ligament for recurrent lateral patellar dislocation. Knee Surg Sports Traumatol Arthrosc. 2014;22(10):2452-7.

53. Deie M, Ochi M, Sumen Y, Adachi N, Kobayashi K, Yasumoto M A long-term follow-up study after medial patellofemoral ligament reconstruction using the transferred semitendinosus tendon for patellar dislocation. Knee Surg Sports Traumatol Arthrosc. 2005;13(7):522-8.

54. Nomura E, Inoue M. Hybrid medial patellofemoral ligament reconstruction using the semitendinous tendon for recurrent patellar dislocation: minimum 3 years' follow-up. Arthroscopy. 2006;22(7):787-93.

55. Ostermeier S, Stukenborg-Colsman C, Wirth CJ, Bohnsack M. [Reconstruction of the medial patellofemoral ligament by tunnel transfer of the semitendinosus tendon]. Oper Orthop Traumatol. 2007;19(5-6):489-501.

56. Steensen RN, Dopirak RM, Maurus PB. A simple technique for reconstruction of the medial patellofemoral ligament using a quadriceps tendon graft. Arthroscopy. 2005;21(3):365-70.

57. Sillanpaa PJ, Maenpaa HM, Mattila VM, Visuri T, Pihlajamaki H. A mini-invasive adductor magnus tendon transfer technique for medial patellofemoral ligament reconstruction: a technical note. Knee Surg Sports Traumatol Arthrosc. 2009;17(5):508-12.

58. Avikainen VJ, Nikku RK, Seppanen-Lehmonen TK. Adductor magnus tenodesis for patellar dislocation. Technique and preliminary results. Clin Orthop Relat Res. 1993;(297):12-6.

59. Trillat A, Dejour H, Couette A. [Diagnosis and Treatment of Recurrent Dislocations of the Patella]. Rev ChirOrthop Reparatrice Appar Mot. 1964;50:813-24.

60. Caton JH, Dejour D. Tibial tubercle osteotomy in patello-femoral instability and in patellar height abnormality. Int Orthop. 2010;34(2):305-9.

61. Ntagiopoulos PG, Byn P, Dejour D. Midterm results of comprehensive surgical reconstruction including sulcus-deepening trochleoplasty in recurrent patellar dislocations with highgrade trochlear dysplasia. Am J Sports Med. 2013;41(5):998-1004. 